\title{
Serum Level of Homocysteine, Folate and Vitamin-B12 in Epileptic Patients Under Carbamazepine and Sodium Valproate Treatment: A Systematic Review and Meta-Analysis
}

\author{
Fazel Gorjipour ${ }^{1}$, Yasin Asadi ${ }^{1,2}$, Nushin K. Osguei ${ }^{3}$, Marjan Effatkhah ${ }^{4}$, Ali Samadikuchak- \\ saraei $^{5,6, *}$ \\ ${ }^{1}$ Physiology Research Center, Iran University of Medical Sciences, Hemmat Campus, Tehran, IR Iran \\ 2 Physiology Research Center, Semnan University of Medical Sciences, Semnan, IR Iran \\ ${ }^{3}$ Eposcience Millennium Institute, Tehran, IR Iran \\ ${ }^{4}$ Department of Neurology, Faculty of Medicine, Iran University of Medical Sciences, Tehran, IR Iran \\ ${ }^{5}$ Department of Medical Biotechnology, Faculty of Allied Medicine, Iran University of Medical Sciences, Tehran, IR Iran \\ ${ }^{6}$ Cellular and Molecular Research Center, Iran University of Medical Sciences, Tehran, IR Iran \\ *Corresponding author: Ali Samadikuchaksaraei, Department of Medical Biotechnology, Faculty of Allied Medicine, Tehran University of Medical Sciences, \\ P.O Box: 14155/6183, Tehran, IR Iran. Tel: +98-2188052984, Fax: +98-2188054355, E-mail: samadikuchaksaraei@yahoo.com.
}

\begin{abstract}
A B S T R A C T
Background: Numerous studies have shown that long term treatment with anticonvulsants may be an important risk factor for the onset of atherosclerosis, or worsening of its symptoms. There are many contradictory reports regarding these effects.

objectives: We performed a systematic review and meta-analysis of the published studies in order to see whether the atherogenic outcomes could be related to any serum biochemical abnormalities.

Materials and Methods: Published articles indexed in PubMed, ISI web of science, Science Direct and Scopus databases from 1990 to 2011 were retrieved using a comprehensive search strategy. After omitting the unrelated articles and duplicates, articles met the eligibility criteria for critical appraisal were included in the analysis. Data were summarized in standard data abstraction forms and subjected to analysis by STATA software.

Results: Finally, ten published studies were included in the meta-analysis. Results showed that carbamazepine and sodium valproate consumption are associated with a significant elevation of the serum homocysteine levels. On the other hand, medication with carbamazepine is associated with a reduction of the level of folate in the serum and that of sodium valproate is associated with a reduction of serum level of vitamin B12.

Conclusions: According to the results of this study, as carbamazepine and valproate sodium consumption can result in elevated serum levels of homocysteine and decreased levels of folate and vitamin B12, and the atherogenic effect of increased serum homocysteine level is well established, the patients under these medications should be monitored for possible atherogenic effects.
\end{abstract}

Keywords: Epilepsy; Anticonvulsants; Atherosclerosis; Adverse Effects; Carbamazepine; Valproic Acid

Copyright @ 2013, Iranian Red Crescent Medical Journal; Published by Kowsar Corp.

Article type: Research Article; Received: 18 Dec 2012, Revised: 08 Jan 2013, Accepted: 26 Feb 2013; DOI: 10.5812/ircmj.9690

PPlease cite this paper as:

Gorjipour F, Asadi Y, K. Osguei N, Effatkhah M, Samadikuchaksaraei A. Serum Level of Homocysteine, Folate and Vitamin-B12 in Epileptic Patients Under Carbamazepine and Sodium Valproate Treatment: A Systematic Review and Meta-Analysis. Iran Red Cres J. 2013;15(3): 249-53. DOI: 10.5812/ircmj.9690

-Implication for health policy/practice/research/medical sciences:

Numerous people are suffering from seizure and are under treatment with anticonvulsants. Results of the current study explore the changes in markers associated with the risk of developing atherosclerosis as reported in the literature. According to our results, the patients under treatment with two common anticonvulsants, carbamazepine and sodium valproate, should be monitored for possible atherogenic effects.

Copyright (C) 2013, Iranian Red Crescent Medical Journal; Published by Kowsar Corp.

This is an Open Access article distributed under the terms of the Creative Commons Attribution License (http://creativecommons.org/licenses/by/3.0), which permits unrestricted use, distribution, and reproduction in any medium, provided the original work is properly cited. 


\section{Background}

Epilepsy is a major neurologic disorder which affects about five to ten percent of the people worldwide. It is a chronic and dynamic medical condition which requires long term, and usually lifelong, treatment with anticonvulsants (1). Epidemiological studies have shown that in adults suffering from epilepsy, risk of development of atherogenic ischemic heart disease (IHD) and fatal cardiovascular disease increase to $34 \%$ and $68 \%$, respectively (2). The underlying etiology of atherosclerosis-related vascular disease in epileptic patients has not yet been fully addressed. The possible etiologic suggestions are factors like alterations in the homocysteine metabolisms, lipid profile aberrations, serum lipoprotein increment, and deficiency of thyroidal hormones $(2,3)$. Impairment of endothelial function is another possible mechanism, which has been presumed to be the result of epilepsy itself, or side effects of long term treatment with antiepileptic agents. This may induce the process of atherogenesis and result in arterial obstructive diseases leading to stroke, myocardial infarction, etc. (4). Among the serum biochemical changes, alterations of the serum homocysteine, folate, and B12 following anticonvulsant therapy and their relationship to atherosclerosis is a subject of controversy (5-8)as the metabolism of these biochemical factors are inter-related and deficiency of either folate or vitamin B12 increases the serum homocysteine level.

\section{Objectives}

In the current study, we attempted to perform a systematic review and meta-analysis to find out whether there is a relationship between anticonvulsant therapy and alterations of serum levels of homocysteine, folate and vitamin B12 as an underlying cause of increased risk of atherogenicity in patients receiving anticonvulsant therapy.

\section{Materials and Methods}

\subsection{Search Strategy and Databases}

In this systematic review and meta-analysis, we first retrieved all articles published from 1990 to 2011 in PubMed, ISI Web of Science, Scopus and Science Direct databases. Keywords included carbamazepine, sodium valproate, epilepsy, antiepileptic, anticonvulsant, folate, folic acid, vitamin B12, homocystein and atherosclerosis. For PubMed we also used the MeSH controlled vocabulary to improve the specificity of our search strategy. For other databases simple searches based on keywords in title and abstract was used. Language of search strategy was limited to English. After exclusion of duplicate results, publications from animal studies, and abstracts presented in scientific meetings, the articles were reviewed by two independent researchers, and those related to this study were chosen. Data was extracted from selected publications and recorded in standard data abstraction forms.
Finally two independent researchers chose the articles which were to be included in the analysis according to the critical appraisal criteria. If there was any ambiguity in inclusion of a study, the opinion of a third reviewer would be sought. Observational studies on patients on antiepileptic medications, in which mean and standard deviation of serum homocysteine, folate and vitamin B12 were reported, and their full-texts were available in English were included in the analysis. Articles not in English or those not reporting the mean and standard deviation homocysteine, folate and vitamin B12 were excluded from study. If there was any ambiguity about the reported results, the authors were directly contacted for clarification. Data related to the type of study, date of study, age group of subjects, gender of subjects, country in which the research was conducted, number of subjects, drugs, outcome measures, and reported results were entered into a spreadsheet file and analyzed by STATA v.11 (College Station, TX, Stata Corporation).

\subsection{Data analysis}

For analysis, standardized mean difference (SMD) of extracted data, and the mean command in the STATA software were used. Also, the Cochrane's Q test for assessment of studies heterogeneity and funnel plot and Egger's regression method for evaluation of the publication bias were used. The significance level was set at $\mathrm{P}<0.05$. This study is a secondary research and does not include ethical codes for human samples. Accordingly, approval of the research ethics committee was not necessary for this study. However, the researchers were committed to follow all of the standards and regulations related to research ethics.

\section{Results}

We retrieved 145 articles from PubMed, 341 articles from ISI Web of Knowledge, 528 articles from Scopus, and 46 articles from Science Direct. To sum up 1060 articles were retrieved and imported into EndNote software (v X.5, Thomson Reuters, New York, NY) where all duplicate results were discarded. Unrelated articles, conference abstracts and animal studies were deleted from the library. Thirty two articles were selected for a full-text assessment. After primary assessment of full-text articles, eight were omitted from the search results as they were unrelated to the topic or were review or case reports. Twenty four articles were thoroughly assessed again. In this step, 11 studies were excluded for the following reasons: the data of more than one antiepileptic agent was pooled, the mean or standard deviation was not reported and the study was comparing the effects of different antiepileptic (Figure 1). Three cohort studies were also excluded as their follow-up durations were different (20 weeks, 32 weeks and 12 months) and it was not possible to analyze their data. Finally, 10 studies were included in the analysis (Table 1). All included studies were of case-control type (Table 
1). Results of meta-analysis and forest plots of the effect estimates for the relationship between receiving carbamazepine and the serum level of homocysteine, folate and vitamin B12 are represented in Figures 2, 3 and 4. Metaanalysis showed that the level of homocysteine was significantly higher in patients receiving carbamazepine ( $\mathrm{P}$ $<0.001$ ) (P for Heterogeneity $\leq 0.001$; I-squared $=94.6 \%$; no publication bias in funnel plot, no significant Egger's test $[\mathrm{P}>0.05])$, while the serum folate levels were significantly lower than controls $(\mathrm{P}<0.001)$ ( $\mathrm{P}$ for Heterogeneity $\leq 0.001$; I-squared $=94.3 \%$; no publication bias in funnel plot, no significant Egger's test $(P>0.05)$. Although, meta-analysis showed a reduction in the serum level of vitamin B12 in patients on carbamazepine, this reduction was not significant $(\mathrm{P}=0.10)$ (I-squared $=49.8 \%$; $\mathrm{P}$ for Heterogeneity $=0.08$; no publication bias in funnel plot, no significant Egger's test $(\mathrm{P}>0.05)$ (Table 2). In regards to treatment with sodium valproate, the results showed that it was significantly associated with increased serum homocysteine levels $(\mathrm{P}<0.001)(\mathrm{p}$ for heterogeneity $=$ 0.003 ; I-squared $=72.1 \%$; no publication bias in funnel plot, no significant Egger's test [P $>0.05])$, but was not associated with reduction of the serum folate levels $(\mathrm{P}=$ 0.52 ) ( $p$ for heterogeneity $=0.01$; I-squared $=65.7 \%$; no publication bias in funnel plot, no significant Egger's test [P $>0.05])$. On the other hand, a significant decrease in the serum levels of vitamin B12 was observed in patients receiving this medication ( $\mathrm{P} \leq 0.001)$ ( $\mathrm{p}$ for heterogeneity $=0.2$; I-squared $=31.9 \%$; no publication bias in funnel plot, no significant Egger's test $(\mathrm{P}>0.05)$ (Table 2).

\section{Discussion}

Cardiovascular atherosclerotic diseases are amongst the most important causes of mortality worldwide. Although clinical manifestations do not occur until adulthood, pathologic changes start from childhood. Implication of the blood lipids aberrations in the pathogenesis of atherosclerosis is well-established $(16,17)$. Atherosclerosis is a multifactorial process. Possible prognostic factors include hyperlipidemia, high blood pressure, hyperglycemia, vitamins B6, B12 and folate de- ficiency and hyperhomocysteinaemia $(18,19)$. Homocysteine is a sulfur containing amino acid produced from metabolism of methionine, an essential amino acid. Elevated serum levels of this amino acid is a strong and independent predictor for increased risk of atherosclerosis development in coronary, aortic, carotid and peripheral arteries in a dose-dependent manner (20).

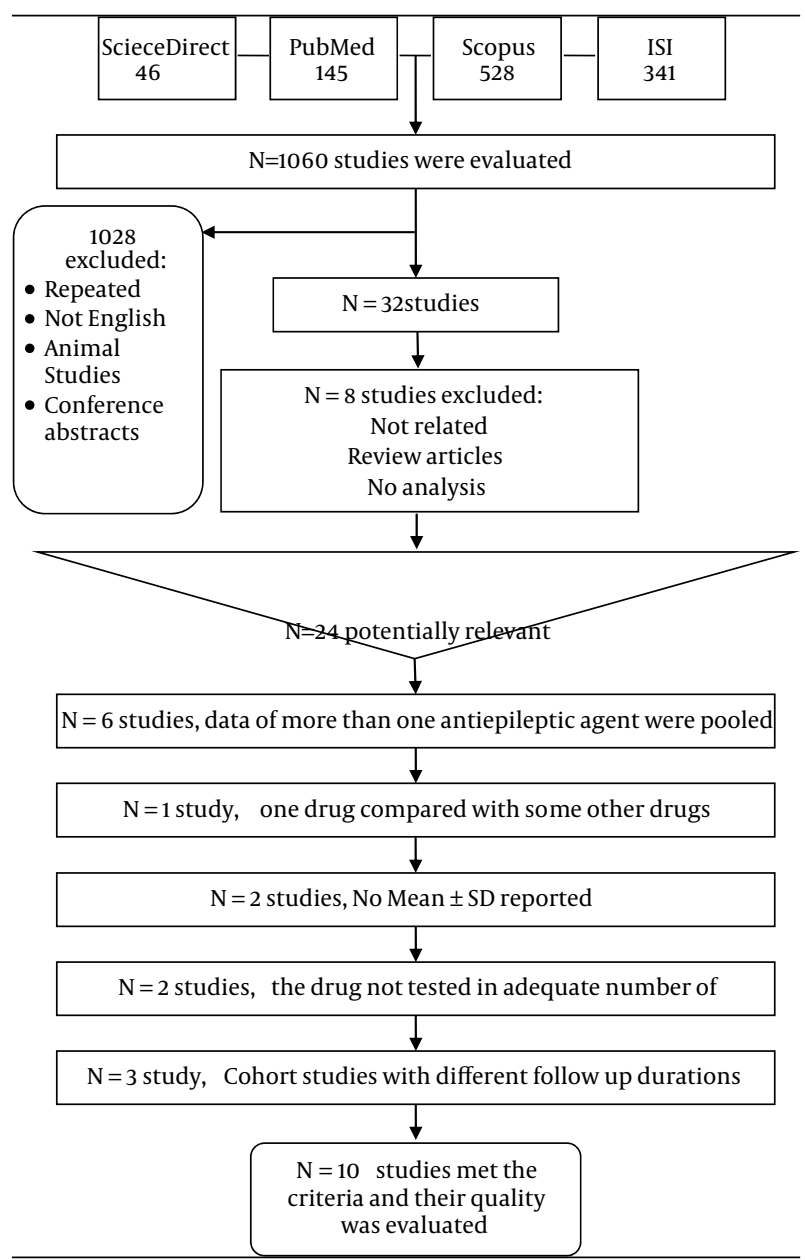

Figure 1. Flowchart depicts the process of selection of studies to be included in this analysis

\begin{tabular}{|c|c|c|c|c|c|c|c|c|}
\hline References & Study & Country & Year & Study Type & Sample Size & Control & Gender & Age, $y$ \\
\hline (9) & Hamed & Egypt & 2007 & $\mathrm{CC}^{\mathrm{a}}$ & 225 & 60 & Male-Female & $18-45$ \\
\hline (10) & Schwaninger & Germany & 1999 & $\mathrm{CC}$ & 51 & 51 & Male-Female & $49.1 \pm 13.9^{a}$ \\
\hline (6) & Karabiber & Turkey & 2003 & CC & 66 & 29 & Male-Female & $2-16$ \\
\hline (3) & Yildiz & Turkey & 2010 & CC & 59 & 23 & Male-Female & $4-13$ \\
\hline (11) & Deda & Turkey & 2003 & CC & 16 & 16 & Male-Female & $5-19$ \\
\hline (12) & Sener & Turkey & 2006 & CC & 75 & 11 & Male-Female & $16-56$ \\
\hline (7) & Kurul & Turkey & 2007 & CC & 25 & 10 & Male-Female & $6-18$ \\
\hline (13) & Hasan & Syria & 2010 & CC & 45 & 28 & Male-Female & $6-64$ \\
\hline (14) & Algin & Turkey & 2009 & CC & 42 & 30 & Male-Female & $45.8 \pm 3.4^{b}$ \\
\hline (15) & Geda & Turkey & 2002 & $\mathrm{CC}$ & 26 & 28 & Male-Female & $1-12$ \\
\hline
\end{tabular}

a Abbreviation: $\mathrm{CC}$, case control

$\mathrm{b}$ This study presented the age as the Mean \pm SD and did not mention the age range of the patients 
Table 2. Results of the Meta-Analysis for Studies Reporting the Effects of Carbamazepine and Sodium Valproate on the Atherosclerosis Risk Factors

\begin{tabular}{lllllll}
\hline AED & Serum Level & Study, No. & SMD $^{\mathrm{a}}$ & $\mathbf{9 5 \% \text { Conf. }}$ & Interval & Pvalue \\
\hline CBZ $^{\mathrm{a}}$ & Homocys $^{\mathrm{a}}$ & 7 & 1.54 & 1.30 & 1.77 & $<0.001$ \\
& Folate & 7 & -1.34 & -1.60 & -1.07 & $<0.001$ \\
& B12 & 6 & -0.22 & -0.48 & 0.04 & 0.10 \\
Na Val $^{\mathrm{a}}$ & Homocys & 6 & 0.76 & 0.51 & 1.02 & $<0.001$ \\
& Folate & 6 & -0.09 & -0.35 & 0.17 & 0.52 \\
& B12 & 6 & 0.57 & 0.30 & 0.83 & $<0.001$ \\
\hline
\end{tabular}

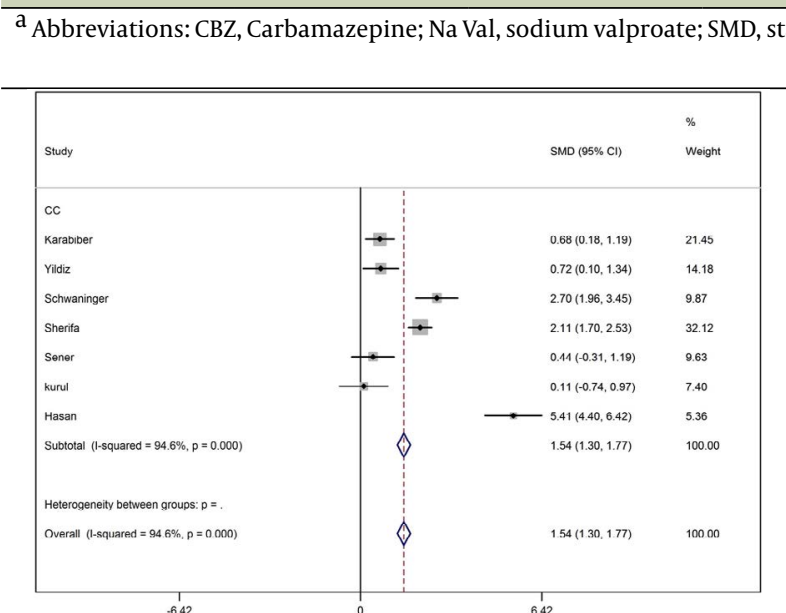

Figure 2 Forest Plot Representing the Effects of Carbamazepine on Blood Homocysteine Level

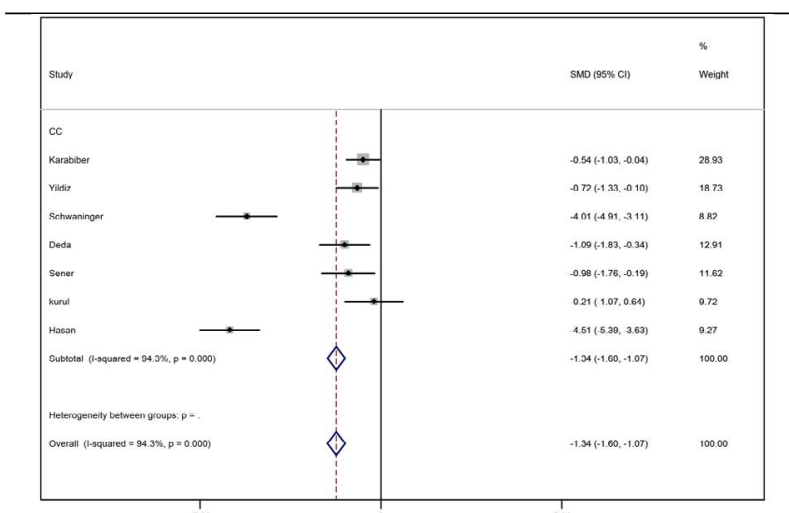

Figure 3. Forest Plot Representing the Effect of Carbamazepine on BloodFolate Level

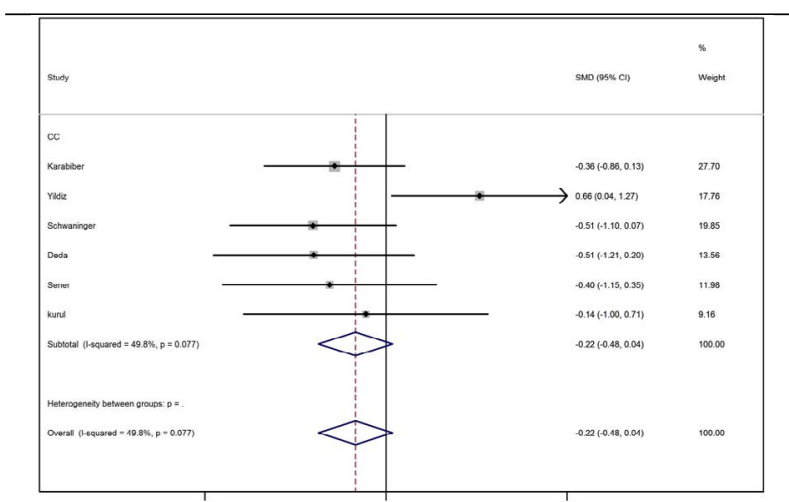

Figure 4. Forest Plot Representing the Effect of Carbamazepine on Blood Vitamin B12 Level
The current study shows that receiving carbamazepine and sodium valproate is significantly associated with increased serum levels of homocysteine, which could be considered as the underlying cause of atherogenicity with consumption of these drugs. Since vitamins B6, B12 and folate are necessary for the metabolism of homocysteine to methionine; their deficiencies are amongst the most important causes of hyperhomocysteinemia. This might be the mechanism by which carbamazepine and sodium valproate increase the serum homocysteine level. On the other hand, it has been mentioned that some antiepileptics can lead to alterations of serum biochemicals such as cholesterol, lipoprotein and homocysteine through induction of cytochrome P450 (21). Changes in the serum level of thyroid hormones have also been mentioned as one of the possible mechanisms of atherogenicity of antiepileptic agents $(2,4,22-24)$. However, the data collected from the articles evaluated in the current study did not consider serum lipids and thyroid hormone levels in a way suitable for inclusion in meta-analysis study. It should be noted that the majority of publications included in this study are from Turkey indicating that there is a high interest in this country to study variations of serum biochemical factors resulting from antiepileptic therapy. The low publication bias indices of this study confirm that no particular bias was involved in inclusion of publications from Turkey. However, more conclusive results could be drawn if measurements of the serum levels of homocysteine, folate and vitamin B12 following antiepileptic therapy could be conducted in populations with more diverse geographical and ethnical background. According to our results, the atherogenic effects of homocysteine and the association of hyperhomocysteinemia with the treatment of carbamazepine and sodium valproate indicate thatthese antiepileptics should be considered atherogenic. It is reasonable to carefully monitor patients receiving these drugs for potential atherogenic effects. Further prospective studies with adequate sample sizes are necessary to consolidate the results from this study.

\section{Acknowledgements}

This study is supported by Tehran University of Medical Sciences (TUMS). 


\section{Authors' Contribution}

Fazel Gorjipour has contributed in all major parts of the study including the design of the study, designing search strategy and search, analysis and manuscript preparation. Marjan Effatkhah has contributed in retrieving and selection of studies to be included in the analysis and data abstraction. Nushin $\mathrm{K}$ Osguei has contributed in the study by helping with study selection and statistical analysis. Ali Samadikuchaksaraei supervised this project, contributed in study design, statistical analysis and manuscript preparation. He also finally reviewed the manuscript for publication.

\section{Financial Disclosure}

Authors declare no financial competing interests.

\section{Funding Support}

None declared.

\section{References}

1. Tan TY, Lu CH, Chuang HY, Lin TK, Liou CW, Chang WN, et al. Longterm antiepileptic drug therapy contributes to the acceleration of atherosclerosis. Epilepsia.2009;50(6):1579-86

2. Jakubus T, Michalska-Jakubus M, Lukawski K, Janowska A, Czuczwar SJ. Atherosclerotic risk among children taking antiepileptic drugs. Pharmacol Rep.2009;61(3):411-23

3. Yildiz M, Simsek G, Uzun H, Uysal S, Sahin S, Balci H. Assessment of low-density lipoprotein oxidation, paraoxonase activity, and arterial distensibility in epileptic children who were treated with anti-epileptic drugs. Cardiol Young.2010;20(5):547-54

4. Hamed SA, Nabeshima T. The high atherosclerotic risk among epileptics: the atheroprotective role of multivitamins. J Pharmacol Sci.2005;98(4):340-53

5. Algin DI, Erdinc OO, Alatas O, Toker A, Colak O, Oner S. Effects of oxcarbazepine on plasma homocysteine, vitamin B12, Folic Acid levels. Noropsikiyatri Ars.2009;46(2):49-51

6. Karabiber H, Sonmezgoz E, Ozerol E, Yakinci C, Otlu B, Yologlu S. Effects of valproate and carbamazepine on serum levels of homocysteine, vitamin B12, and folic acid. Brain Dev.2003;25(2):113-5

7. Kurul S, Unalp A, Yis U. Homocysteine levels in epileptic children receiving antiepileptic drugs. J Child Neurol.2007;22(12):1389-92

8. Zeitlhofer J, Doppelbauer A, Tribl G, Leitha T, Deecke L. Changes of serum lipid patterns during long-term anticonvulsive treatment. Clin Investig.1993;71(7):574-8

9. Hamed SA, Hamed EA, Hamdy R, Nabeshima T. Vascular risk factors and oxidative stress as independent predictors of asymptomatic atherosclerosis in adult patients with epilepsy. Epilepsy
Res.2007;74(2-3):183-92

10. Schwaninger M, Ringleb P, Winter R, Kohl B, Fiehn W, Rieser PA, et al. Elevated plasma concentrations of homocysteine in antiepileptic drug treatment. Epilepsia.1999;40(3):345-50

11. Deda G, Caksen H, Icagasioglu D. Effect of long-term carbamazepine therapy on serum lipids, vitamin B12 and folic acid levels in children.J Pediatr Endocrinol Metab.2003;16(2):193-6

12. Sener U, Zorlu Y, Karaguzel O, Ozdamar O, Coker I, Topbas M. Effects of common anti-epileptic drug monotherapy on serum levels of homocysteine, vitamin B12, folic acid and vitamin B6. Seizure.2006;15(2):79-85

13. Hasan K, Saleh Y, Assaad F. Effects of carbamazepine on serum levels of folic acid and homocysteine. Pan Arab J Neurosurg.2010;14(1):108-11

14. Algin DI, Erdinç OO, Alatas, Ö. , Toker, A. , Colak, Ö. , Öner, S. . Effects of oxcarbazepine on plasma homocysteine, vitamin B12, folic acid levels. Noropsikiyatri Arsivi.2009;;46(2):49-51

15. Geda G, Caksen H, Icagasioglu D. Serum lipids, vitamin B12 and folic acid levels in children receiving long-term valproate therapy. Acta Neurol Belg.2002;102(3):122-6

16. Fallah S, Nouroozi V, Seifi M, Samadikuchaksaraei A, Aghdashi EM. Influence of oral contraceptive pills on homocysteine and nitric oxide levels: as risk factors for cardiovascular disease. J Clin Lab Anal.2012;26(2):120-3

17. Norouzi V, Seifi M, Fallah S, Korani M, Samadikuchaksaraei A Effect of oral contraceptive therapy on homocysteine and C-reactive protein levels in women: an observational study. Anadolu Kardiyol Derg.2011;11(8):698-702

18. Jellinger PS, Smith DA, Mehta AE, Ganda O, Handelsman Y, Rodbard HW, et al. American Association of Clinical Endocrinologists' Guidelines for Management of Dyslipidemia and Prevention of Atherosclerosis. Endocr Pract.2012;18 Suppl 1:1-78

19. Perk J, De Backer G, Gohlke H, Graham I, Reiner Z, Verschuren WM, et al. European Guidelines on cardiovascular disease prevention in clinical practice (version 2012): The Fifth Joint Task Force of the European Society of Cardiology and Other Societies on Cardiovascular Disease Prevention in Clinical Practice (constituted by representatives of nine societies and by invited experts). Atherosclerosis.2012;223(1):1-68

20. Guthikonda S, Haynes WG. Homocysteine: role and implications in atherosclerosis. Curr Atheroscler Rep.2006;8(2):100-6

21. Lopinto-Khoury C, Mintzer S. Antiepileptic drugs and markers of vascular risk. Curr Treat Options Neurol.2010;12(4):300-8

22. Eiris J, Novo-Rodriguez MI, Del Rio M, Meseguer P, Del Rio MC, Castro-Gago M. The effects on lipid and apolipoprotein serum levels of long-term carbamazepine, valproic acid and phenobarbital therapy in children with epilepsy. Epilepsy Res.2000;41(1):1-7

23. Verrotti A, Domizio S, Angelozzi B, Sabatino G, Morgese G, Chiarelli F. Changes in serum lipids and lipoproteins in epileptic children treated with anticonvulsants. I Paediatr Child Health.1997;33(3):242-5

24. Yilmaz E, Dosan Y, Gurgoze MK, Gungor S. Serum lipid changes during anticonvulsive treatment serum lipids in epileptic children. Acta Neurol Belg.2001;101(4):217-20 